\title{
Extending the applicability of a third-order scheme with Lipschitz and Hölder continuous derivative in Banach spaces
}

\author{
Debasis Sharma* (D) and Sanjaya Kumar Parhi
}

\section{*Correspondence:}

iiit.debasis@gmail.com

Department of Mathematics,

International Institute of

Information Technology

Bhubaneswar, Odisha, 751003, India

\begin{abstract}
We extend the applicability of a cubically convergent nonlinear system solver using Lipschitz continuous first-order Fréchet derivative in Banach spaces. This analysis avoids the usual application of Taylor expansion in convergence analysis and extends the applicability of the scheme by applying the technique based on the first-order derivative only. Also, our study provides the radius of convergence ball and computable error bounds along with the uniqueness of the solution. Furthermore, the generalization of this analysis using Hölder condition is provided. Various numerical tests confirm that our analysis produces better results and it is useful in solving such problems where previous methods can not be implemented.
\end{abstract}

Keywords: Local convergence, Iterative schemes, Banach space, Lipschitz continuity condition, Hölder continuity condition

AMS Subject Classification (2010): 47H99; 49M15; 65J15; 65D99; 65G99

\section{Introduction}

The main aim of this paper is to extend the applicability of a third-order nonlinear system solver for estimating a locally unique solution $s^{*}$ of

$$
G(s)=0,
$$

where $G: \Omega \subseteq X \rightarrow Y$ is a Fréchet differentiable operator with values in the Banach space $Y$ and $\Omega$ is a convex subset of the Banach space $X$. In the domain of engineering and applied sciences, plenty of problems are solved by converting into nonlinear equations of the form (1). For example, the integral equations arise in radiative transfer theory, problems in optimization, several boundary value problems, and many others can be transformed into the equations in the form (1). For solving these equations, the most commonly used technique is iterative algorithms. A popular and widely accepted iterative procedure for solving (1) is Newton's algorithm, which is expressed as:

$$
s_{k+1}=s_{k}-\left[G^{\prime}\left(s_{k}\right)\right]^{-1} G\left(s_{k}\right), k \geq 0 .
$$

(C) The Author(s). 2020 Open Access This article is licensed under a Creative Commons Attribution 4.0 International License which permits use, sharing, adaptation, distribution and reproduction in any medium or format, as long as you give appropriate credit to the original author(s) and the source, provide a link to the Creative Commons licence, and indicate if changes were made. The images or other third party material in this article are included in the article's Creative Commons licence, unless indicated otherwise in a credit line to the material. If material is not included in the article's Creative Commons licence and your intended use is not permitted by statutory regulation or exceeds the permitted use, you will need to obtain permission directly from the copyright holder. To view a copy of this licence, visit http://creativecommons.org/licenses/by/4.0/. 
Some classical cubically convergent schemes like Chebyshev's, Super-Halley's, and the Halley's algorithms are generated by substituting $(\beta=0),(\beta=1)$ and $\left(\beta=\frac{1}{2}\right)$ respectively in

$$
s_{k+1}=s_{k}-\left(1+\frac{1}{2}\left(1-\beta H_{G}\left(s_{k}\right)\right)^{-1} H_{G}\left(s_{k}\right)\right)\left[G^{\prime}\left(s_{k}\right)\right]^{-1} G\left(s_{k}\right),
$$

where $H_{G}\left(s_{k}\right)=G^{\prime}\left(s_{k}\right)^{-1} G^{\prime \prime}\left(s_{k}\right) G^{\prime}\left(s_{k}\right)^{-1} G\left(s_{k}\right)$.

Convergence analysis is an important part in the study of iterative methods. This is generally categorized into two types, viz. semi-local and local convergence. "The semi-local convergence is based on the information around an initial point and gives criteria that ensures the convergence of iteration procedures". "The local convergence analysis of iterative schemes is based on the information around a solution and provides the radius of convergence ball as well as the convergence domain"[1]. "The choice of the initial point is a shot in the dark, in general since the radius of convergence is not computed. The radius of convergence is useful even in cases when the solution is not known. Also, the local analysis provides computable error bounds as well as the uniqueness of the solution" [2]. Numerous researchers [1,3-5] studied the local convergence analysis of different variants of Chebyshev-Halley type schemes including modified Halley-like, deformed Halley, and improved Chebyshev-Halley type methods. In addition to that, the local convergence study for Newton-type, Jarratt-type, Weerakoon-type, etc., is studied in Banach spaces in $[2,6-15]$. In this study, our primary focus is to enhance the applicability of a thirdorder scheme using Lipschitz continuity condition only on first-order Fréchet derivative in Banach spaces.

In [16], the authors derived a third-order algorithm applying quadrature rule for obtaining the solutions of nonlinear systems. The scheme is given as:

$$
\begin{aligned}
t_{k} & =s_{k}-\left[G^{\prime}\left(s_{k}\right)\right]^{-1} G\left(s_{k}\right) \\
s_{k+1} & =s_{k}-4\left[G^{\prime}\left(s_{k}\right)+3 G^{\prime}\left(\frac{1}{3}\left(s_{k}+2 t_{k}\right)\right)\right]^{-1} G\left(s_{k}\right)
\end{aligned}
$$

To execute this scheme, we require the computation of the first-order Fréchet derivative. However, the analysis of convergence is established by applying the Taylor series approach based on higher-order derivatives. These techniques, which need the higher-order derivatives, limit the applicability of the algorithm. As an illustration, define a function $G$ on $\Omega=\left[-\frac{1}{2}, \frac{5}{2}\right]$ by

$$
G(s)= \begin{cases}s^{3} \log \left(s^{2}\right)+s^{5}-s^{4}, & \text { if } \quad s \neq 0 \\ 0, & \text { if } \quad s=0\end{cases}
$$

It is important to note that $G^{\prime \prime \prime}$ is unbounded on $\Omega$. Therefore, the theory based on higher-order derivatives [16] fail to solve the above problem. Also, one can get no idea about the domain of convergence in [16]. The local convergence study gives valuable information regarding the radius of convergence ball. For the algorithm (4), we discuss the local convergence by following the approach based on $G^{\prime}$ to stay away from the evaluation of higher-order Fréchet derivatives. In particular, we consider that the first-order Fréchet derivative belongs to the Lipschitz class. This analysis enlarges the utility of the method (4) by solving such problems for which earlier studies can not be used due to the computation higher-order Fréchet derivatives. 
We arrange the manuscript as follows: the "Local convergence analysis" section deals with the local convergence study of the algorithm (4). The "Numerical examples" is dedicated to the numerical applications of our analytical results. The "Conclusions" section is placed in the last section.

\section{Local convergence analysis}

We describe the local convergence analysis of the algorithm (4) in this segment. Let the notations for the closed and open balls with center $c$ and radius $\rho>0$ be $\bar{B}(c, \rho)$ and $B(c, \rho)$ respectively. $B L(Y, X)$ is the notation for the set of all bounded linear operators from $Y$ to $X$. $k_{0}, k_{1}$ be two positive parameters with $k_{0} \leq k_{1}$. In Theorems 1 and 2, we provide the local convergence analysis of the scheme (4).

Local convergence analysis of the method (4) under Lipschitz continuity condition

We define the function $\Phi_{1}$ on the interval $\left[0, \frac{1}{k_{0}}\right)$ by

$$
\Phi_{1}(u)=\frac{k_{1} u}{2\left(1-k_{0} u\right)}
$$

and the parameter

$$
\eta_{1}=\frac{2}{2 k_{0}+k_{1}}<\frac{1}{k_{0}} .
$$

It is easy to observe that $\Phi_{1}\left(\eta_{1}\right)=1$. Again, we define the functions $\Phi_{2}$ and $\Psi_{2}$ on $\left[0, \frac{1}{k_{0}}\right)$ by

$$
\Phi_{2}(u)=\frac{1}{3}\left(1+2 \Phi_{1}(u)\right)
$$

and

$$
\Psi_{2}(u)=\Phi_{2}(u)-1 .
$$

Now, $\Psi_{2}(0)=-\frac{2}{3}<0$ and $\lim \Psi_{2}(u)=+\infty$. The intermediate value theorem con-

$$
u \rightarrow\left(\frac{1}{k_{0}}\right)^{-}
$$

firms the existence of the zeros of the function $\Psi_{2}(u)$ in $\left(0, \frac{1}{k_{0}}\right)$. We denote the smallest zero of $\Psi_{2}(u)$ in $\left(0, \frac{1}{k_{0}}\right)$ as $\eta_{2}$. Again, we define $\Phi_{3}$ and $\Psi_{3}$ on $\left[0, \frac{1}{k_{0}}\right)$ by

$$
\Phi_{3}(u)=\frac{k_{0}}{4}\left(1+3 \Phi_{2}(u)\right) u
$$

and

$$
\Psi_{3}(u)=\Phi_{3}(u)-1 .
$$

Now, $\Psi_{3}(0)=-1<0$ and $\lim _{u \rightarrow\left(\frac{1}{k_{0}}\right)^{-}} \Psi_{3}(u)=+\infty$. So, The zeros of the function $\Psi_{3}(u)$ lies in $\left(0, \frac{1}{k_{0}}\right)$. We denote the smallest zero of $\Psi_{3}(u)$ in $\left(0, \frac{1}{k_{0}}\right)$ as $\eta_{3}$. Lastly, we define $\Phi_{4}$ and $\Psi_{4}$ on $\left[0, \eta_{3}\right)$ by

$$
\Phi_{4}(u)=\frac{k_{1}\left[1+\frac{3}{2} \Phi_{2}(u)\right] u}{2\left(1-\Phi_{3}(u)\right)}
$$

and

$$
\Psi_{4}(u)=\Phi_{4}(u)-1 .
$$


Now, $\Psi_{4}(0)=-1<0$ and $\lim _{u \rightarrow \eta_{3}^{-}} \Psi_{4}(u)=+\infty$. Let $\eta_{4}$ be the notation for the smallest zero of $\Psi_{4}(u)$ in $\left(0, \eta_{3}\right)$. The existence of $\eta_{4}$ is guaranteed by the intermediate value theorem. We choose

$$
R^{\prime}=\min \left\{\eta_{1}, \eta_{2}, \eta_{4}\right\}
$$

to confirm the followings.

$$
\begin{aligned}
& 0 \leq \Phi_{1}(u)<1, \\
& 0 \leq \Phi_{2}(u)<1, \\
& 0 \leq \Phi_{3}(u)<1
\end{aligned}
$$

and

$$
0 \leq \Phi_{4}(u)<1,
$$

for each $u \in\left[0, R^{\prime}\right)$. Also, we use the following assumptions on the Fréchet differentiable operator $G: \Omega \subseteq X \rightarrow Y$.

$$
\begin{array}{r}
G\left(s^{*}\right)=0, G^{\prime}\left(s^{*}\right)^{-1} \in B L(Y, X), \\
\left\|G^{\prime}\left(s^{*}\right)^{-1}\left(G^{\prime}(s)-G^{\prime}\left(s^{*}\right)\right)\right\| \leq k_{0}\left\|s-s^{*}\right\|, \forall s \in \Omega
\end{array}
$$

and

$$
\left\|G^{\prime}\left(s^{*}\right)^{-1}\left(G^{\prime}(s)-G^{\prime}(t)\right)\right\| \leq k_{1}\|s-t\|, \forall s, t \in \Omega .
$$

Now, we discuss the local convergence analysis of the algorithm (4) in Theorem 1.

Theorem 1 Let $s^{*} \in \Omega$. Suppose the Fréchet differentiable operator $G: \Omega \subseteq X \rightarrow Y$ obeys (14)-(16) and

$$
\bar{B}\left(s^{*}, R^{\prime}\right) \subseteq \Omega,
$$

where $R^{\prime}$ is given in (9). Starting from $s_{0} \in B\left(s^{*}, R^{\prime}\right)$ the scheme (4) produces the sequence $\left\{s_{k}\right\}$ which is well defined, $\left\{s_{k}\right\}_{k \geq 0} \in B\left(s^{*}, R^{\prime}\right)$ and converges to $s^{*}$. Also, the followings hold $\forall k \geq 0$

$$
\begin{aligned}
& \left\|t_{k}-s^{*}\right\| \leq \Phi_{1}\left(\left\|s_{k}-s^{*}\right\|\right)\left\|s_{k}-s^{*}\right\|<\left\|s_{k}-s^{*}\right\|<R^{\prime} \\
& \left\|\left[G^{\prime}\left(s_{k}\right)+3 G^{\prime}\left(\frac{1}{3}\left(s_{k}+2 t_{k}\right)\right)\right]^{-1} G^{\prime}\left(s^{*}\right)\right\| \leq \frac{1}{4\left(1-\Phi_{3}\left(\left\|s_{k}-s^{*}\right\|\right)\right)}
\end{aligned}
$$

and

$$
\left\|s_{k+1}-s^{*}\right\| \leq \Phi_{4}\left(\left\|s_{k}-s^{*}\right\|\right)\left\|s_{k}-s^{*}\right\|<\left\|s_{k}-s^{*}\right\|<R^{\prime},
$$

where the functions $\Phi_{1}, \Phi_{3}$, and $\Phi_{4}$ are provided in (5), (7), and (8) respectively. For $\delta \in$ $\left[R^{\prime}, \frac{2}{k_{0}}\right)$, the equation $G(s)=0$ has only one solution $s^{*}$ in $\bar{B}\left(s^{*}, \delta\right) \cap \Omega$.

Proof It follows from (9), (15) and the assumption $s_{0} \in B\left(s^{*}, R^{\prime}\right)$ that

$$
\left\|G^{\prime}\left(s^{*}\right)^{-1}\left(G^{\prime}\left(s_{0}\right)-G^{\prime}\left(s^{*}\right)\right)\right\| \leq k_{0}\left\|s_{0}-s^{*}\right\|<k_{0} R^{\prime}<1 .
$$

Now, Banach Lemma on invertible operators [17-21] ensures that $G^{\prime}\left(s_{0}\right)^{-1} \in B L(Y, X)$ and

$$
\left\|G^{\prime}\left(s_{0}\right)^{-1} G^{\prime}\left(s^{*}\right)\right\| \leq \frac{1}{1-k_{0}\left\|s_{0}-s^{*}\right\|}<\frac{1}{1-k_{0} R^{\prime}} .
$$


Therefore, $t_{0}$ is well defined. Again,

$$
\begin{aligned}
t_{0}-s^{*} & =s_{0}-s^{*}-G^{\prime}\left(s_{0}\right)^{-1} G\left(s_{0}\right) \\
& =-\left[G^{\prime}\left(s_{0}\right)^{-1} G^{\prime}\left(s^{*}\right)\right]\left[\int_{0}^{1} G^{\prime}\left(s^{*}\right)^{-1}\left(G^{\prime}\left(s^{*}+\theta\left(s_{0}-s^{*}\right)\right)-G^{\prime}\left(s_{0}\right)\right)\left(s_{0}-s^{*}\right) d \theta\right] .
\end{aligned}
$$

Using (5), (9), (10), (16), (22) and (23), we find

$$
\begin{aligned}
\left\|t_{0}-s^{*}\right\| & \leq\left[\left\|G^{\prime}\left(s_{0}\right)^{-1} G^{\prime}\left(s^{*}\right)\right\|\right]\left[\left\|\int_{0}^{1} G^{\prime}\left(s^{*}\right)^{-1}\left(G^{\prime}\left(s^{*}+\theta\left(s_{0}-s^{*}\right)\right)-G^{\prime}\left(s_{0}\right)\right)\left(s_{0}-s^{*}\right) d \theta\right\|\right] \\
& \leq \frac{k_{1}\left\|s_{0}-s^{*}\right\|}{2\left(1-k_{0}\left\|s_{0}-s^{*}\right\|\right)}\left\|s_{0}-s^{*}\right\| \\
& =\Phi_{1}\left(\left\|s_{0}-s^{*}\right\|\right)\left\|s_{0}-s^{*}\right\|<\left\|s_{0}-s^{*}\right\|<R^{\prime} .
\end{aligned}
$$

So, (18) holds for $k=0$. Now,

$$
\begin{aligned}
\left\|\frac{1}{3}\left(s_{0}+2 t_{0}\right)-s^{*}\right\| & \leq \frac{1}{3}\left\|\left(s_{0}+2 t_{0}\right)-3 s^{*}\right\| \\
& \leq \frac{1}{3}\left(\left\|s_{0}-s^{*}\right\|+2\left\|t_{0}-s^{*}\right\|\right) \\
& \leq \frac{1}{3}\left(\left\|s_{0}-s^{*}\right\|+2 \Phi_{1}\left(\left\|s_{0}-s^{*}\right\|\right)\left\|s_{0}-s^{*}\right\|\right) \\
& =\frac{1}{3}\left(1+2 \Phi_{1}\left(\left\|s_{0}-s^{*}\right\|\right)\right)\left\|s_{0}-s^{*}\right\| \\
& =\Phi_{2}\left(\left\|s_{0}-s^{*}\right\|\right)\left\|s_{0}-s^{*}\right\|<\left\|s_{0}-s^{*}\right\|<R^{\prime} .
\end{aligned}
$$

So, $\frac{1}{3}\left(s_{0}+2 t_{0}\right) \in B\left(s^{*}, R^{\prime}\right)$. Then, our claim is $\left[G^{\prime}\left(s_{0}\right)+3 G^{\prime}\left(\frac{1}{3}\left(s_{0}+2 t_{0}\right)\right)\right]^{-1} \in B L(Y, X)$. The equations (7), (9), (12), (15), (24), and (25) are used to deduce

$$
\begin{aligned}
& \left\|\left(4 G^{\prime}\left(s^{*}\right)\right)^{-1}\left[G^{\prime}\left(s_{0}\right)+3 G^{\prime}\left(\frac{1}{3}\left(s_{0}+2 t_{0}\right)\right)-4 G^{\prime}\left(s^{*}\right)\right]\right\| \\
& \leq \frac{1}{4}\left[\left\|G^{\prime}\left(s^{*}\right)^{-1}\left(G^{\prime}\left(s_{0}\right)-G^{\prime}\left(s^{*}\right)\right)\right\|+3\left\|G^{\prime}\left(s^{*}\right)^{-1}\left(G^{\prime}\left(\frac{1}{3}\left(s_{0}+2 t_{0}\right)\right)-G^{\prime}\left(s^{*}\right)\right)\right\|\right] \\
& \leq \frac{k_{0}}{4}\left[\left\|s_{0}-s^{*}\right\|+3\left\|\frac{1}{3}\left(s_{0}+2 t_{0}\right)-s^{*}\right\|\right] \\
& \leq \frac{k_{0}}{4}\left[\left\|s_{0}-s^{*}\right\|+3 \Phi_{2}\left(\left\|s_{0}-s^{*}\right\|\right)\left\|s_{0}-s^{*}\right\|\right] \\
& =\frac{k_{0}}{4}\left[1+3 \Phi_{2}\left(\left\|s_{0}-s^{*}\right\|\right)\right]\left\|s_{0}-s^{*}\right\| \\
& =\Phi_{3}\left(\left\|s_{0}-s^{*}\right\|\right)<\Phi_{3}\left(R^{\prime}\right)<1 .
\end{aligned}
$$

Now, we obtain $\left[G^{\prime}\left(s_{0}\right)+3 G^{\prime}\left(\frac{1}{3}\left(s_{0}+2 t_{0}\right)\right)\right]^{-1} \in B L(Y, X)$ using Banach Lemma on invertible operators. Also,

$$
\left\|\left[G^{\prime}\left(s_{0}\right)+3 G^{\prime}\left(\frac{1}{3}\left(s_{0}+2 t_{0}\right)\right)\right]^{-1} G^{\prime}\left(s^{*}\right)\right\| \leq \frac{1}{4\left(1-\Phi_{3}\left(\left\|s_{0}-s^{*}\right\|\right)\right)} .
$$


Hence, $s_{1}$ is well defined. We use (8) (9), (13), (16), (25), and (26) to derive

$$
\begin{aligned}
\left\|s_{1}-s^{*}\right\| & \leq\left(\left\|\left[G^{\prime}\left(s_{0}\right)+3 G^{\prime}\left(\frac{1}{3}\left(s_{0}+2 t_{0}\right)\right)\right]^{-1} G^{\prime}\left(s^{*}\right)\right\|\right) \\
& \left(\left\|\int_{0}^{1} G^{\prime}\left(s^{*}\right)^{-1}\left(G^{\prime}\left(s_{0}\right)-G^{\prime}\left(s^{*}+\theta\left(s_{0}-s^{*}\right)\right)\right)\left(s_{0}-s^{*}\right) d \theta\right\|\right. \\
& \left.+3\left\|\int_{0}^{1} G^{\prime}\left(s^{*}\right)^{-1}\left(G^{\prime}\left(\frac{1}{3}\left(s_{0}+2 t_{0}\right)\right)-G^{\prime}\left(s^{*}+\theta\left(s_{0}-s^{*}\right)\right)\right)\left(s_{0}-s^{*}\right) d \theta\right\|\right) \\
& \leq \frac{\frac{k_{1}}{2}\left\|s_{0}-s^{*}\right\|^{2}+3 k_{1} \int_{0}^{1}\left(\left\|\frac{1}{3}\left(s_{0}+2 t_{0}\right)-s^{*}-\theta\left(s_{0}-s^{*}\right)\right\|\right) d \theta\left\|s_{0}-s^{*}\right\|}{4\left(1-\Phi_{3}\left(\left\|s_{0}-s^{*}\right\|\right)\right)} \\
& \leq \frac{\frac{k_{1}}{2}\left\|s_{0}-s^{*}\right\|^{2}+3 k_{1}\left(\left\|\frac{1}{3}\left(s_{0}+2 t_{0}\right)-s^{*}\right\|+\frac{\left\|s_{0}-s^{*}\right\|}{2}\right)\left\|s_{0}-s^{*}\right\|}{4\left(1-\Phi_{3}\left(\left\|s_{0}-s^{*}\right\|\right)\right)} \\
& \leq \frac{\frac{k_{1}}{2}\left\|s_{0}-s^{*}\right\|^{2}+3 k_{1}\left[\Phi_{2}\left(\left\|s_{0}-s^{*}\right\|\right)\left\|s_{0}-s^{*}\right\|+\frac{\left\|s_{0}-s^{*}\right\|}{2}\right]\left\|s_{0}-s^{*}\right\|}{4\left(1-\Phi_{3}\left(\left\|s_{0}-s^{*}\right\|\right)\right)} \\
= & \frac{\left(2 k_{1}\left\|s_{0}-s^{*}\right\|+3 k_{1} \Phi_{2}\left(\left\|s_{0}-s^{*}\right\|\right) \| s_{0}-s^{*}||\right)\left\|s_{0}-s^{*}\right\|}{4\left(1-\Phi_{3}\left(\left\|s_{0}-s^{*}\right\|\right)\right)} \\
= & \frac{k_{1}\left[\left(1+\frac{3}{2} \Phi_{2}\left(\left\|s_{0}-s^{*}\right\|\right)\right)\left\|s_{0}-s^{*}\right\|\right]\left\|s_{0}-s^{*}\right\|}{2\left(1-\Phi_{3}\left(\left\|s_{0}-s^{*}\right\|\right)\right)} \\
= & \Phi_{4}\left(\left\|s_{0}-s^{*}\right\|\right)\left\|s_{0}-s^{*}\right\|<\left\|s_{0}-s^{*}\right\|<R^{\prime} .
\end{aligned}
$$

Therefore, we prove that (20) is true for $k=0$. We find the estimates (18)-(20) by substituting $s_{k}, t_{k}$ and $s_{k+1}$ in place of $s_{0}, t_{0}$ and $s_{1}$ respectively in the earlier estimations. From the inequality $\left\|s_{k+1}-s^{*}\right\| \leq \Phi_{4}\left(R^{\prime}\right)\left\|s_{k}-s^{*}\right\|<R^{\prime}$, we have $s_{k+1} \in B\left(s^{*}, R^{\prime}\right)$ and $\lim _{k \rightarrow \infty} s_{k}=s^{*}$. Now, we have to show the uniqueness part. Let $t^{*}\left(\neq s^{*}\right) \in B\left(s^{*}, \delta\right)$ be such that $0=G\left(t^{*}\right)$. Consider $B=\int_{0}^{1} G^{\prime}\left(t^{*}+\theta\left(s^{*}-t^{*}\right)\right) d \theta$. From Eq. (15), we get

$$
\begin{aligned}
\left\|G^{\prime}\left(s^{*}\right)^{-1}\left(B-G^{\prime}\left(s^{*}\right)\right)\right\| & \leq \int_{0}^{1} k_{0}\left\|t^{*}+\theta\left(s^{*}-t^{*}\right)-s^{*}\right\| d \theta \\
& \leq \frac{k_{0}}{2}\left\|s^{*}-t^{*}\right\| \\
& \leq \frac{k_{0} \delta}{2}<1 .
\end{aligned}
$$

Applying Banach Lemma, we confirm that $B^{-1} \in B L(Y, X)$. The identity $0=G\left(s^{*}\right)-$ $G\left(t^{*}\right)=B\left(s^{*}-t^{*}\right)$ implies that $s^{*}=t^{*}$.

\section{Local convergence analysis of the method (4) under Hölder continuity condition}

There are numerous problems for which the technique based on Lipschitz condition fails to solve without using higher-order derivatives. As an illustration, we consider the following problem given in [6].

$$
G(s)(x)=s(x)-3 \int_{0}^{1} G_{1}(x, y) s(y)^{\frac{5}{4}} d y,
$$

where $s(x) \in C[0,1]$ and $G_{1}(x, y)$ is Green's function defined on $[0,1] \times[0,1]$ by

$$
G_{1}(x, y)=\left\{\begin{array}{l}
(1-x) y, \text { if } y \leq x \\
x(1-y), \text { if } x \leq y
\end{array}\right.
$$


Then,

$$
\left\|G^{\prime}(s)-G^{\prime}(t)\right\| \leq \frac{15}{32}\|s-t\|^{\frac{1}{4}} .
$$

It is important to note that $G^{\prime}$ does not obey Lipschitz condition. However, $G^{\prime}$ is Hölder continuous. So, we discuss the local convergence of the method (4) with Hölder continuous first-order Fréchet derivative. This analysis also generalizes the local convergence analysis presented in the previous section.

For $q \in(0,1]$, we define $\Theta_{1}$ on $\left[0,\left(\frac{1}{k_{0}}\right)^{\frac{1}{q}}\right)$ by

$$
\Theta_{1}(w)=\frac{k_{1} w^{q}}{(q+1)\left(1-k_{0} w^{q}\right)}
$$

and the parameter

$$
\xi_{1}=\left(\frac{(q+1)}{(q+1) k_{0}+k_{1}}\right)^{\frac{1}{q}}<\left(\frac{1}{k_{0}}\right)^{\frac{1}{q}} .
$$

Observe that $\Theta_{1}\left(\xi_{1}\right)=1$. Again, we define functions $\Theta_{2}$ and $\Gamma_{2}$ on $\left[0,\left(\frac{1}{k_{0}}\right)^{\frac{1}{q}}\right)$ by

$$
\Theta_{2}(w)=\frac{1}{3}\left(1+2 \Theta_{1}(w)\right)
$$

and

$$
\Gamma_{2}(w)=\Theta_{2}(w)-1 .
$$

Now, $\Gamma_{2}(0)=-\frac{2}{3}<0$ and $\quad \lim \Gamma_{2}(w)=+\infty$. The intermediate value theorem $w \rightarrow\left(\left(\frac{1}{k_{0}}\right)^{\frac{1}{q}}\right)^{-}$

confirms the existence of the zeros of the function $\Gamma_{2}(w)$ in $\left(0,\left(\frac{1}{k_{0}}\right)^{\frac{1}{q}}\right)$. We denote the smallest zero of $\Gamma_{2}(w)$ in $\left(0,\left(\frac{1}{k_{0}}\right)^{\frac{1}{q}}\right)$ as $\xi_{2}$. Again, we define $\Theta_{3}$ and $\Gamma_{3}$ on $\left[0,\left(\frac{1}{k_{0}}\right)^{\frac{1}{q}}\right)$ by

$$
\Theta_{3}(w)=\frac{k_{0}}{4}\left[1+3 \Theta_{2}(w)^{q}\right] w^{q}
$$

and

$$
\Gamma_{3}(w)=\Theta_{3}(w)-1 .
$$

Now, $\Gamma_{3}(0)=-1<0$ and $\quad \lim \Gamma_{3}(w)=+\infty$. So, the zeros of the function $\Gamma_{3}(w)$ $w \rightarrow\left(\left(\frac{1}{k_{0}}\right)^{\frac{1}{q}}\right)^{-}$

lies in $\left(0,\left(\frac{1}{k_{0}}\right)^{\frac{1}{q}}\right)$. We denote the smallest zero of $\Gamma_{3}(w)$ in $\left(0,\left(\frac{1}{k_{0}}\right)^{\frac{1}{q}}\right)$ as $\xi_{3}$. Lastly, we define $\Theta_{4}$ and $\Gamma_{4}$ on $\left[0, \xi_{3}\right)$ by

$$
\Theta_{4}(w)=\frac{k_{1}\left(\frac{4}{q+1}+3 \Theta_{2}(w)^{q}\right) w^{q}}{4\left(1-\Theta_{3}(w)\right)}
$$

and

$$
\Gamma_{4}(w)=\Theta_{4}(w)-1 .
$$

Now, $\Gamma_{4}(0)=-1<0$ and $\lim _{w \rightarrow \xi_{3}^{-}} \Gamma_{4}(w)=+\infty$. Let $\xi_{4}$ be the notation for the smallest zero of $\Gamma_{4}(w)$ in $\left(0, \xi_{3}\right)$. The existence of $\xi_{4}$ is guaranteed by the intermediate value theorem. We choose 


$$
R=\min \left\{\xi_{1}, \xi_{2}, \xi_{4}\right\}
$$

to confirm the followings.

$$
\begin{aligned}
& 0 \leq \Theta_{1}(w)<1, \\
& 0 \leq \Theta_{2}(w)<1, \\
& 0 \leq \Theta_{3}(w)<1
\end{aligned}
$$

and

$$
0 \leq \Theta_{4}(w)<1
$$

for each $w \in[0, R)$. Also, we use the following assumptions on the Fréchet differentiable operator $G: \Omega \subseteq X \rightarrow Y$.

$$
\begin{array}{r}
G\left(s^{*}\right)=0, G^{\prime}\left(s^{*}\right)^{-1} \in B L(Y, X), \\
\left\|G^{\prime}\left(s^{*}\right)^{-1}\left(G^{\prime}(s)-G^{\prime}\left(s^{*}\right)\right)\right\| \leq k_{0}\left\|s-s^{*}\right\|^{q}, \forall s \in \Omega
\end{array}
$$

and

$$
\left\|G^{\prime}\left(s^{*}\right)^{-1}\left(G^{\prime}(s)-G^{\prime}(t)\right)\right\| \leq\left. k_{1}\|s-t\|\right|^{q}, \forall s, t \in \Omega .
$$

Theorem 2 Let $s^{*} \in \Omega$. Suppose the Fréchet differentiable operator $G: \Omega \subseteq X \rightarrow Y$ obeys (37)-(39) and

$$
\bar{B}\left(s^{*}, R\right) \subseteq \Omega,
$$

where $R$ is given in (32). Starting from $s_{0} \in B\left(s^{*}, R\right)$, the scheme (4) yields the sequence $\left\{s_{k}\right\}$ which is well defined, $\left\{s_{k}\right\}_{k \geq 0} \in B\left(s^{*}, R\right)$ and converges to $s^{*}$. Also, the following holds $\forall k \geq 0$

$$
\begin{array}{r}
\left\|t_{k}-s^{*}\right\| \leq \Theta_{1}\left(\left\|s_{k}-s^{*}\right\|\right)\left\|s_{k}-s^{*}\right\|<\left\|s_{k}-s^{*}\right\|<R, \\
\left\|\left[G^{\prime}\left(s_{k}\right)+3 G^{\prime}\left(\frac{1}{3}\left(s_{k}+2 t_{k}\right)\right)\right]^{-1} G^{\prime}\left(s^{*}\right)\right\| \leq \frac{1}{4\left(1-\Theta_{3}\left(\left\|s_{k}-s^{*}\right\|\right)\right)}
\end{array}
$$

and

$$
\left\|s_{k+1}-s^{*}\right\| \leq \Theta_{4}\left(\left\|s_{k}-s^{*}\right\|\right)\left\|s_{k}-s^{*}\right\|<\left\|s_{k}-s^{*}\right\|<R,
$$

where the functions $\Theta_{1}, \Theta_{3}$, and $\Theta_{4}$ are provided in (28), (30), and (31) respectively. For $\Delta \in\left[R,\left(\frac{q+1}{k_{0}}\right)^{\frac{1}{q}}\right)$, the solution $s^{*}$ is the only solution of $G(s)=0$ in $\bar{B}\left(s^{*}, \Delta\right) \cap \Omega$.

Proof It follows from (32), (38) and the assumption $s_{0} \in B\left(s^{*}, R\right)$ that

$$
\left\|G^{\prime}\left(s^{*}\right)^{-1}\left(G^{\prime}\left(s_{0}\right)-G^{\prime}\left(s^{*}\right)\right)\right\| \leq k_{0}\left\|s_{0}-s^{*}\right\|^{q}<k_{0} R^{q}<1 .
$$

Now, Banach Lemma on invertible operators [17-21] ensures that $G^{\prime}\left(s_{0}\right)^{-1} \in B L(Y, X)$ and

$$
\left\|G^{\prime}\left(s_{0}\right)^{-1} G^{\prime}\left(s^{*}\right)\right\| \leq \frac{1}{1-k_{0}\left\|s_{0}-s^{*}\right\|^{q}}<\frac{1}{1-k_{0} R^{q}} .
$$


Therefore, $t_{0}$ is well defined. Again,

$$
\begin{aligned}
t_{0}-s^{*} & =s_{0}-s^{*}-G^{\prime}\left(s_{0}\right)^{-1} G\left(s_{0}\right) \\
& =-\left[G^{\prime}\left(s_{0}\right)^{-1} G^{\prime}\left(s^{*}\right)\right]\left[\int_{0}^{1} G^{\prime}\left(s^{*}\right)^{-1}\left(G^{\prime}\left(s^{*}+\theta\left(s_{0}-s^{*}\right)\right)-G^{\prime}\left(s_{0}\right)\right)\left(s_{0}-s^{*}\right) d \theta\right] .
\end{aligned}
$$

Using (28), (32), (33), (39), (45) and (46), we find

$$
\begin{aligned}
\left\|t_{0}-s^{*}\right\| & \leq\left[\left\|G^{\prime}\left(s_{0}\right)^{-1} G^{\prime}\left(s^{*}\right)\right\|\right]\left[\left\|\int_{0}^{1} G^{\prime}\left(s^{*}\right)^{-1}\left(G^{\prime}\left(s^{*}+\theta\left(s_{0}-s^{*}\right)\right)-G^{\prime}\left(s_{0}\right)\right)\left(s_{0}-s^{*}\right) d \theta\right\|\right] \\
& \leq \frac{k_{1}\left\|s_{0}-s^{*}\right\|^{q}}{(q+1)\left(1-k_{0} \| s_{0}-s^{*}||^{q}\right)}\left\|s_{0}-s^{*}\right\| \\
& =\Theta_{1}\left(\left\|s_{0}-s^{*}\right\|\right)\left\|s_{0}-s^{*}\right\|<\left\|s_{0}-s^{*}\right\|<R .
\end{aligned}
$$

So, (41) holds for $k=0$. Now,

$$
\begin{aligned}
\left\|\frac{1}{3}\left(s_{0}+2 t_{0}\right)-s^{*}\right\| & \leq \frac{1}{3}\left\|\left(s_{0}+2 t_{0}\right)-3 s^{*}\right\| \\
& \leq \frac{1}{3}\left(\left\|s_{0}-s^{*}\right\|+2\left\|t_{0}-s^{*}\right\|\right) \\
& \leq \frac{1}{3}\left(\left\|s_{0}-s^{*}\right\|+2 \Theta_{1}\left(\left\|s_{0}-s^{*}\right\|\right)\left\|s_{0}-s^{*}\right\|\right) \\
& =\frac{1}{3}\left(1+2 \Theta_{1}\left(\left\|s_{0}-s^{*}\right\|\right)\right)\left\|s_{0}-s^{*}\right\| \\
& =\Theta_{2}\left(\left\|s_{0}-s^{*}\right\|\right)\left\|s_{0}-s^{*}\right\|<\left\|s_{0}-s^{*}\right\|<R .
\end{aligned}
$$

So, $\frac{1}{3}\left(s_{0}+2 t_{0}\right) \in B\left(s^{*}, R\right)$. Then, our claim is $\left[G^{\prime}\left(s_{0}\right)+3 G^{\prime}\left(\frac{1}{3}\left(s_{0}+2 t_{0}\right)\right)\right]^{-1} \in B L(Y, X)$. The Eqs. (30), (32), (35), (38), (47), and (48) are used to deduce

$$
\begin{aligned}
& \left\|\left(4 G^{\prime}\left(s^{*}\right)\right)^{-1}\left[G^{\prime}\left(s_{0}\right)+3 G^{\prime}\left(\frac{1}{3}\left(s_{0}+2 t_{0}\right)\right)-4 G^{\prime}\left(s^{*}\right)\right]\right\| \\
& \leq \frac{1}{4}\left[\left\|G^{\prime}\left(s^{*}\right)^{-1}\left(G^{\prime}\left(s_{0}\right)-G^{\prime}\left(s^{*}\right)\right)\right\|+3\left\|G^{\prime}\left(s^{*}\right)^{-1}\left(G^{\prime}\left(\frac{1}{3}\left(s_{0}+2 t_{0}\right)\right)-G^{\prime}\left(s^{*}\right)\right)\right\|\right] \\
& \leq \frac{k_{0}}{4}\left[\left\|s_{0}-s^{*}\right\|^{q}+3\left\|\frac{1}{3}\left(s_{0}+2 t_{0}\right)-s^{*}\right\|^{q}\right] \\
& \leq \frac{k_{0}}{4}\left[\left\|s_{0}-s^{*}\right\|^{q}+3 \Theta_{2}\left(\left\|s_{0}-s^{*}\right\|\right)^{q}\left\|s_{0}-s^{*}\right\|^{q}\right] \\
& =\frac{k_{0}}{4}\left[1+3 \Theta_{2}\left(\left\|s_{0}-s^{*}\right\|\right)^{q}\right]\left\|s_{0}-s^{*}\right\|^{q} \\
& =\Theta_{3}\left(\left\|s_{0}-s^{*}\right\|\right)<\Theta_{3}(R)<1 .
\end{aligned}
$$

Now, we obtain $\left[G^{\prime}\left(s_{0}\right)+3 G^{\prime}\left(\frac{1}{3}\left(s_{0}+2 t_{0}\right)\right)\right]^{-1} \in B L(Y, X)$ using Banach Lemma on invertible operators. Also,

$$
\left\|\left[G^{\prime}\left(s_{0}\right)+3 G^{\prime}\left(\frac{1}{3}\left(s_{0}+2 t_{0}\right)\right)\right]^{-1} G^{\prime}\left(s^{*}\right)\right\| \leq \frac{1}{4\left(1-\Theta_{3}\left(\left\|s_{0}-s^{*}\right\|\right)\right)} .
$$

Table 1 Parameters for example 1

\begin{tabular}{lllll}
\hline Algorithm (4) & & & & Midpoint method [10] \\
\cline { 1 - 3 }$\xi_{1}$ & $\xi_{2}$ & $\xi_{4}$ & $R$ & $r$ \\
\hline 0.324947 & 0.324947 & 0.241437 & 0.241437 & 0.085221 \\
\hline
\end{tabular}


Table 2 Parameters for example 2

\begin{tabular}{lllll}
\hline Algorithm (4) & & & & Midpoint method [10] \\
\cline { 1 - 3 }$\xi_{1}$ & $\xi_{2}$ & $\xi_{4}$ & $R$ & $r$ \\
\hline 0.666667 & 0.666667 & 0.542573 & 0.542573 & 0.333333 \\
\hline
\end{tabular}

Hence, $s_{1}$ is well defined. We use (31) (32), (36), (39), (48), and (49) to derive

$$
\begin{aligned}
& \left\|s_{1}-s^{*}\right\| \leq\left(\left\|\left[G^{\prime}\left(s_{0}\right)+3 G^{\prime}\left(\frac{1}{3}\left(s_{0}+2 t_{0}\right)\right)\right]^{-1} G^{\prime}\left(s^{*}\right)\right\|\right) \\
& \left(\left\|\int_{0}^{1} G^{\prime}\left(s^{*}\right)^{-1}\left(G^{\prime}\left(s_{0}\right)-G^{\prime}\left(s^{*}+\theta\left(s_{0}-s^{*}\right)\right)\right)\left(s_{0}-s^{*}\right) d \theta\right\|\right. \\
& \left.+3\left\|\int_{0}^{1} G^{\prime}\left(s^{*}\right)^{-1}\left(G^{\prime}\left(\frac{1}{3}\left(s_{0}+2 t_{0}\right)\right)-G^{\prime}\left(s^{*}+\theta\left(s_{0}-s^{*}\right)\right)\right)\left(s_{0}-s^{*}\right) d \theta\right\|\right) \\
& \leq \frac{\frac{k_{1}}{q+1}\left\|s_{0}-s^{*}\right\|^{q+1}+3 k_{1} \int_{0}^{1}\left(\left.\left\|\frac{1}{3}\left(s_{0}+2 t_{0}\right)-s^{*}-\theta\left(s_{0}-s^{*}\right)\right\|\right|^{q}\right) d \theta\left\|s_{0}-s^{*}\right\|}{4\left(1-\Theta_{3}\left(\left\|s_{0}-s^{*}\right\|\right)\right)} \\
& \leq \frac{\frac{k_{1}}{q+1}\left\|s_{0}-s^{*}||^{q+1}+3 k_{1}\left(\| \frac{1}{3}\left(s_{0}+2 t_{0}\right)-s^{*}||^{q}+\frac{\left\|s_{0}-s^{*}\right\|^{q}}{q+1}\right)\right\| s_{0}-s^{*} \|}{4\left(1-\Theta_{3}\left(\left\|s_{0}-s^{*}\right\|\right)\right)} \\
& \leq \frac{\frac{k_{1}}{q+1}\left\|s_{0}-s^{*}||^{q+1}+3 k_{1}\left[\Theta_{2}\left(\left\|s_{0}-s^{*}\right\|\right)^{q}\left\|s_{0}-s^{*}\right\|^{q}+\frac{\| s_{0}-s^{*}||^{q}}{q+1}\right]\right\| s_{0}-s^{*} \|}{4\left(1-\Theta_{3}\left(\left\|s_{0}-s^{*}\right\|\right)\right)} \\
& =\frac{\left(\frac{k_{1}}{q+1}\left\|s_{0}-s^{*}\right\|^{q}+3 k_{1}\left[\Theta_{2}\left(\| s_{0}-s^{*}||\right)^{q}\left\|s_{0}-s^{*}\right\|^{q}+\frac{\| s_{0}-s^{*}||^{q}}{q+1}\right]\right)\left\|s_{0}-s^{*}\right\|}{4\left(1-\Theta_{3}\left(\left\|s_{0}-s^{*}\right\|\right)\right)} \\
& =\frac{\left(\frac{4 k_{1}}{q+1} \| s_{0}-s^{*}||^{q}+3 k_{1}\left[\Theta_{2}\left(\| s_{0}-s^{*}||\right)^{q}\left\|s_{0}-s^{*}\right\|^{q}\right]\right)\left\|s_{0}-s^{*}\right\|}{4\left(1-\Theta_{3}\left(\left\|s_{0}-s^{*}\right\|\right)\right)} \\
& =\frac{k_{1}\left(\left.\frac{4}{q+1}\left\|s_{0}-s^{*}\right\|\right|^{q}+3\left[\left.\Theta_{2}\left(\| s_{0}-s^{*}||\right)^{q}\left\|s_{0}-s^{*}\right\|\right|^{q}\right]\right)\left\|s_{0}-s^{*}\right\|}{4\left(1-\Theta_{3}\left(\left\|s_{0}-s^{*}\right\|\right)\right)} \\
& =\Theta_{4}\left(|| s_{0}-s^{*}||\right)|| s_{0}-s^{*}\|<\| s_{0}-s^{*} \|<R \text {. }
\end{aligned}
$$

Thus, we prove that (43) holds for $k=0$. We find the estimates (41)-(43) by substituting $s_{k}, t_{k}$, and $s_{k+1}$ in place of $s_{0}, t_{0}$, and $s_{1}$ respectively in the preceding estimations. From the inequality $\left\|s_{k+1}-s^{*}\right\| \leq \Theta_{4}(R)\left\|s_{k}-s^{*}\right\|<R$, we have $s_{k+1} \in B\left(s^{*}, R\right)$ and $\lim _{k \rightarrow \infty} s_{k}=s^{*}$. Now, we have to show the uniqueness part. Let $t^{*}\left(\neq s^{*}\right) \in B\left(s^{*}, \Delta\right)$ be such that $0=$ $G\left(t^{*}\right)$. Consider $A=\int_{0}^{1} G^{\prime}\left(\theta s^{*}+t^{*}(1-\theta)\right) d \theta$. From Eq. (38), we get

$$
\begin{aligned}
\left\|G^{\prime}\left(s^{*}\right)^{-1}\left(A-G^{\prime}\left(s^{*}\right)\right)\right\| & \leq \int_{0}^{1} k_{0}\left\|t^{*}+\theta\left(s^{*}-t^{*}\right)-s^{*}\right\|^{q} d \theta \\
& \leq \frac{k_{0}}{q+1}\left\|s^{*}-t^{*}\right\|^{q} \\
& \leq \frac{k_{0} \Delta^{q}}{q+1}<1 .
\end{aligned}
$$

Table 3 Parameters for example 3

\begin{tabular}{lllll}
\hline Algorithm (4) & & & \multicolumn{2}{l}{ Midpoint method [10] } \\
\cline { 1 - 3 }$\xi_{1}$ & $\xi_{2}$ & $\xi_{4}$ & $R$ & $r$ \\
\hline 0.066667 & 0.066667 & 0.047233 & 0.047233 & 0.019896 \\
\hline
\end{tabular}


Table 4 Parameters for example 4

\begin{tabular}{lllll}
\hline Algorithm (4) & & & \multicolumn{2}{l}{ Midpoint method [10] } \\
\cline { 1 - 3 }$\xi_{1}$ & $\xi_{2}$ & $\xi_{4}$ & $R$ & $r$ \\
\hline 0.006896 & 0.006896 & 0.005613 & 0.005613 & 0.002500 \\
\hline
\end{tabular}

Applying Banach Lemma, we confirm that $A^{-1} \in B L(Y, X)$. The identity $0=G\left(s^{*}\right)-$ $G\left(t^{*}\right)=A\left(s^{*}-t^{*}\right)$ implies that $s^{*}=t^{*}$.

\section{Numerical examples}

In this section, we compute the radii of convergence balls for standard numerical problems. Also, we compare the convergence radii with that of the third order convergent midpoint method obtained by the technique of Argyros and George provided in [10]. We obtain better results using our technique in all cases.

Example 1 [6] Let $G$ is defined on $\bar{B}(0,1)$ for $\left(s_{1}, s_{2}, s_{3}\right)^{t}$ by

$$
G(s)=\left(e^{s_{1}}-1, \frac{e-1}{2} s_{2}^{2}+s_{2}, s_{3}\right)^{t}
$$

We have $s^{*}=(0,0,0)^{t}, q=1, k_{0}=e-1$ and $k_{1}=e$. We compute the value of the radius $R$ employing " $\Theta$ " functions (Table 1).

Example 2 [6] Define $G$ on $\Omega=[-1,1]$ by

$$
G(s)=\sin (s)
$$

We have $s^{*}=0, q=1$ and $k_{0}=k_{1}=1$. R is obtained using " $\Theta$ " functions (Table 2).

Example 3 [7] Consider the nonlinear Hammerstein type integral equation given by

$$
G(s)(x)=s(x)-5 \int_{0}^{1} x y s(y)^{3} d y,
$$

where $G(s) \in C[0,1]$. We have $s^{*}=0, q=1, k_{0}=7.5$ and $k_{1}=15$. We use the definitions of " $\Theta$ " functions to compute the value of $R$ (Table 3).

Example 4 [6] Define $G$ on $\Omega=\left[-\frac{1}{2}, \frac{5}{2}\right]$ by

$$
G(x)= \begin{cases}s^{3} \log \left(s^{2}\right)+s^{5}-s^{4}, & \text { if } s \neq 0 \\ 0, & \text { if } s=0\end{cases}
$$

We have $s^{*}=1, q=1$ and $k_{0}=k_{1}=96.6628$. The radius $R$ is calculated from the definitions of " $\Theta$ " functions.

Thus, we confirm the convergence of the scheme (4) with radius $R=0.005613$ (Table 4).

Table 5 Parameters for example 5

\begin{tabular}{lllll}
\hline Algorithm (4) & & & & \multicolumn{2}{l}{$\begin{array}{l}\text { Midpoint method [10] } \\
\xi_{1}\end{array}$} & $\xi_{2}$ & $\xi_{4}$ & $R$ & $r$ \\
\hline 0.025599 & 0.025599 & 0.014957 & 0.014957 & 0.003887 \\
\hline
\end{tabular}


Table 6 Parameters for example 6

\begin{tabular}{|c|c|c|c|c|}
\hline \multicolumn{4}{|c|}{ Algorithm (4) } & \multirow{2}{*}{$\begin{array}{l}\text { Midpoint method [10] } \\
r\end{array}$} \\
\hline$\xi_{1}$ & $\xi_{2}$ & $\xi_{4}$ & $R$ & \\
\hline 1.973081 & 1.973081 & 0.598292 & 0.598292 & 0.055768 \\
\hline
\end{tabular}

Example 5 [6] Consider the nonlinear Hammerstein type integral equation given by

$$
G(s)(x)=s(x)-5 \int_{0}^{1} x y s(y)^{\frac{3}{2}} d y,
$$

where $s(x) \in C[0,1]$. We have $s^{*}=0$. Also, $q=0.5$ and $k_{0}=k_{1}=\frac{15}{4}$. We use " $\Theta$ " functions to compute the radius $R$ (Table 5).

\section{Example 6 [6] Consider the nonlinear integral equation given by}

$$
G(s)(x)=s(x)-3 \int_{0}^{1} G_{1}(x, y) s(y)^{\frac{5}{4}} d y,
$$

where $s(x) \in C[0,1]$ and $G_{1}(x, y)$ is Green's function. We have $s^{*}=0$. Also, $q=0.25$ and $k_{0}=k_{1}=\frac{15}{32}$. The radius $R$ is computed using " $\Theta$ " functions.

Thus, we guarantee the convergence of the algorithm (4) for example 6 with radius $R=$ 0.598292 (Table 6).

\section{Conclusions}

Local convergence analysis of a higher-order convergent nonlinear system solver (4) is discussed. For expanding the applicability of the method, this study is provided under the only condition that the first-order Fréchet derivative is Lipschitz continuous. This technique is applicable in solving such problems for which previous studies can not be applied. Also, the generalization of this analysis using Hölder condition is studied. At last, standard examples are solved to show the convergence of the scheme.

Acknowledgments

The authors would like to thank the University Grants Commission of India for the financial support.

Authors' contributions

DS raised the first idea and analyzed and wrote the paper. SKP edited and analyzed the paper again in the well-organized form. All authors read and approved the final manuscript.

Funding

Author D. Sharma is financially supported by the University Grants Commission of India (ID: NOV2017-402662) in the form of JRF.

Availability of data and materials

Data sharing not applicable to this article as no datasets were generated or analyzed during the current study.

Competing interests

The authors declare that they have no competing interests.

Received: 20 December 2019 Accepted: 5 May 2020

Published online: 29 May 2020

\section{References}

1. Argyros, I. K., Magreñán, Á. A.: A study on the local convergence and the dynamics of Chebyshev-Halley-type methods free from second derivative. Numer. Algor. 71(1), 1-23 (2015)

2. Sharma, J. R., Argyros, I. K.: Local convergence of a Newton-Traub composition in Banach spaces. SeMA J. 75(1), 57-68 (2017)

3. Argyros, I. K., George, S.: Local convergence of deformed Halley method in Banach space under Hölder continuity conditions. J. Nonlinear Sci. Appl. 8, 246-254 (2015)

4. Argyros, I. K., George, S., Magreñán, Á. A.: Local convergence for multi-point-parametric Chebyshev-Halley-type methods of higher convergence order. J. Comput. Appl. Math. 282, 215-224 (2015) 
5. Argyros, I. K., George, S.: Local convergence of modified Halley-like methods with less computation of inversion. Novi Sad J. Math. 45(2), 47-58 (2015)

6. Singh, S., Gupta, D. K., Badoni, R. P., Martínez, E., Hueso, J. L.: Local convergence of a parameter based iteration with Hölder continuous derivative in Banach spaces. Calcolo. 54(2), 527-539 (2017)

7. Martínez, E., Singh, S., Hueso, J. L., Gupta, D. K.: Enlarging the convergence domain in local convergence studies for iterative methods in Banach spaces. Appl. Math. Comput. 281, 252-265 (2016)

8. Amat, S., Argyros, I. K., Busquier, S., Hernández-Verón, M. A., Martínez, E.: On the local convergence study for an efficient k-step iterative method. J. Comput. Appl. Math. 343, 753-761 (2018)

9. Argyros, I. K., Hilout, S.: On the local convergence of fast two-step Newton-like methods for solving nonlinear equations. J. Comput. Appl. Math. 245, 1-9 (2013)

10. Argyros, I. K., George, S.: Local convergence of two competing third order methods in Banach space. Appl. Math. 41(4), 341-350 (2014)

11. Argyros, I. K., Khattri, S. K.: Local convergence for a family of third order methods in Banach spaces. Appl. Math. Comput. 251, 396-403 (2015)

12. Argyros, I. K., González, D: Local convergence for an improved Jarratt-type method in Banach space. Int. J. Interact. Multimed. Artif. Intell. 3, 20-25 (2015). (Special Issue on Teaching Mathematics Using New and Classic Tools)

13. Argyros, I. K., Cho, Y. J., George, S.: Local convergence for some third order iterative methods under weak conditions. J. Korean Math. Soc. 53(4), 781-793 (2016)

14. Sharma, D., Parhi, S. K.: On the local convergence of modified Weerakoon's method in Banach spaces. J. Anal. (2019). https://doi.org/10.1007/s41478-019-00216-X

15. Cordero, A., Ezquerro, J. A., Hernández-Verón, M. A.: On the local convergence of a fifth-order iterative method in Banach spaces. J. Math. 46, 53-62 (2014)

16. Noor, M. A., Waseem, M.: Some iterative methods for solving a system of nonlinear equations. Comput. Math. Appl. 57, 101-106 (2009)

17. Argyros, I. K.: Convergence and application of Newton-type iterations. Springer, Berlin (2008)

18. Argyros, I. K., Hilout, S.: Computational methods in nonlinear analysis. World Scientific Publishing House, New Jersey (2013)

19. Petković, M. S., Neta, B., Petković, L., Dz̃unić, D.: Multipoint methods for solving nonlinear equations. Elsevier, Amsterdam (2013)

20. Rall, L. B.: Computational solution of nonlinear operator equations (Krieger, R. E., ed.) Krieger Publishing, New York (1979)

21. Traub, J. F.: Iterative methods for solution of equations. Prentice-Hal, Englewood Cliffs (1964)

\section{Publisher's Note}

Springer Nature remains neutral with regard to jurisdictional claims in published maps and institutional affiliations.

\section{Submit your manuscript to a SpringerOpen ${ }^{\circ}$ journal and benefit from:}

- Convenient online submission

Rigorous peer review

- Open access: articles freely available online

- High visibility within the field

- Retaining the copyright to your article

Submit your next manuscript at $\boldsymbol{\triangleright}$ springeropen.com 